\title{
A Multi Platform Pain Assessment Tool using Bespoke Gaming Sensors
}

\author{
Fotios Spyridonis \\ University of Greenwich \\ Old Royal Naval College \\ London, UK \\ $+44(0) 2083318607$ \\ f.spyridonis@greenwich. \\ ac.uk
}

\author{
Tor-Morten Grønli \\ Westerdals Oslo ACT \\ Faculty of Technology \\ Mobile Technology Lab \\ Oslo, Norway \\ +4748156476 \\ tmg@westerdals.no
}

\author{
loannis Th. \\ Paraskevopoulos \\ University of Greenwich \\ Old Royal Naval College \\ London, UK \\ $+44(0) 2083317856$ \\ i.parask@greenwich.ac.uk
}

\author{
Gheorghita Ghinea \\ Brunel University London \\ Uxbridge \\ London, UK \\ $+44(0) 1895266033$ \\ george.ghinea@brunel.ac.uk
}

\begin{abstract}
The use of commercial game devices for the purpose of developing novel interactive solutions in order to support the provision of care has attracted considerable interest over the last years. However, the development of such solutions to use with the clinical assessment of the notoriously challenging experience of pain has fallen behind in recent years. In this paper, we present a proposed conceptual design and one example tool for pain assessment using Microsoft Kinect as the interaction method. Past research demonstrated the usefulness of Virtual Reality, and especially Kinect, in the management and reduction of. It is anticipated that our tool will provide a more natural user experience that enables pain sufferers to also assess their pain experience either in a clinical setting or in the comfort of a home environment.
\end{abstract}

\section{CCS Concepts}

-Human-centered computing Graphical user interfaces -Human-centered computing Virtual reality •Humancentered computing $\sim$ Gestural input $\bullet$ Applied computing Consumer health Applied computing Health care information systems $\bullet$ Hardware $\sim$ Emerging interfaces

\section{Keywords}

Pain; pain drawing; cloud; virtual reality; Kinect; consumer game devices; natural user interfaces; health management

\section{INTRODUCTION}

Pain is typically characterized as "an unpleasant sensory and emotional experience associated with actual or potential tissue damage", or is often described based on such a damage [7]. A pan-European consensus report [1] revealed that one in five Europeans (19\%) is estimated to have some form of pain, and this has had tremendous cost and quality of life implications to healthcare systems across Europe [14][27].

When it comes to managing pain, unlike other vital signs such as blood pressure or heart rate that rely on objective measurements, pain is typically assessed on the basis of a patient's subjective experience. In fact, patient self-reporting has been the most reliable indicator of the existence and intensity of pain [11]. This constitutes an important challenge for healthcare providers, especially considering the multidimensional nature of pain, which

SAMPLE: Permission to make digital or hard copies of all or part of this work for personal or classroom use is granted without fee provided that copies are not made or distributed for profit or commercial advantage and that copies bear this notice and the full citation on the first page. To copy otherwise, or republish, to post on servers or to redistribute to lists, requires prior specific permission and/or a fee.

Petrae Conference'9, Month 6, 2016, City, State, Country. Copyright 2016 ACM 1-58113-000-0/00/0010 ...\$15.00. typically involves physiologic and emotional qualities [13]. It is therefore imperative that self-reporting of pain is effectively embedded into assessment practices and tools.

Conventional intervention practices typically involve a 2dimensional (2D) representation of the human body, i.e. the "pain drawing', which patients use to pinpoint the location and type of pain [5] that are experiencing, as well as a variety of questionnaires and tools [12]. Notwithstanding their advantages, efficient intervention seems to be limited in most cases, with studies indicating only a partial success of past approaches in effectively assessing the pain experience [8][15][16]. This is often accounted by the fact that such experiences are usually described in a paper format, which makes it impractical and subject to error [8], or because they do not capture the 3-dimensional (3D) nature of the human body, e.g. statements of the form "I have a pain on the inside of my thigh" are not easily captured in a $2 \mathrm{D}$ pain drawing. In fact, studies suggest that 2D visualization is not useful anymore for a complete understanding of the 'object' under investigation, mainly because it lacks the natural depth cues (e.g. perspective, shading, and occlusion) [23]. As a result, it is difficult to effectively capture self-reported expressions of pain. Nevertheless, pain assessment today still relies on those practices.

In response, new computerized tools based on Virtual Reality (VR), mobile technology and 3D visualization, to name just a few, have been developed over the last decade to help address the aforementioned issues. The motivation behind leveraging this kind of technologies is related to minimizing consultation times, while at the same time keeping the patient motivated and engaged in his/her own pain management. However, most past efforts focused on using such technology as a means to help patients manage their pain. Past research by the authors revealed that the use of such tools also enables patients to more accurately communicate their pain experience and therefore also help with the assessment process [22]. Moreover, those tools were mostly restricted to graphical user interface (GUI) and specific gesturebased interactions, or relied on small devices, which often are cumbersome to use due to a patient's limited physical movement as a result of their pain.

An alternative is the use of commercial game devices, such as the Nintendo Wii Remote, PlayStation Move or the Microsoft Kinect, which have the potential to enable a reality-like experience while allowing for touch-free interactions. In addition to such platforms, though, users nowadays access content through a variety of sensor-rich devices including smartphones, smart watches, augmented displays - wearables, in one word - in addition to traditional laptops, tablets, personal computers and modern 
Internet-ready TVs. These raise the challenge of allowing users for untethered access to content irrespective of location and device used. To address this issue, in the current paper we propose a platform-agnostic tool for pain assessment centered around cloud-based data access, natural user interfaces and movement interaction.

The remaining of this paper is structured as follows. First, we review existing work in the field, and then we present our conceptual design and tool, followed by a discussion and conclusion.

\section{RELATED WORK}

The leveraging of VR technology towards developing advanced tools for supporting the management of pain has attracted significant attention over the past decade. Hoffman et al. [6], used VR as an adjunct to analgesics for burn pain and demonstrated that this helped in reducing pain and distress. Similarly, in the work of Lamont, Chin and Kogan [10], VR was used as a means of managing phantom limb pain in upper extremity amputees. Their findings showed that VR significantly contributes in the reduction of phantom limb pain. Studies also demonstrated that VR could be effective in decreasing pain in persons with cancer [9].

Other studies focusing on affective interventions showed that VR can be employed as a distraction mechanism, both for chronic pain [24], but also instant pain [4]. For instance, Trost and Parsons [24] devised a VR paradigm with Kinect, exposing sufferers to graded therapy regimes for chronic pain with promising results regarding its applicability, affordability and wide accessibility. Guo, Deng and Yang [4] have utilised VR interventions for instant pain distraction of patients with upper limb injuries undergoing dressing changes with empirical results on the effectiveness of the intervention for both patients and professionals. Another example of how VR can be used for affective interventions is presented by Gromala et al. [3]. The VR meditative walk intervention, as suggested in this study, is found to be more effective to reduce chronic pain perceived by patients, compared to a control group.

Another area that VR has found use in pain related interventions is kinematic assessment for low back pain sufferers. In the study of Roosink et al. [20], a Virtual Mirror is employed for a task specific intervention undertaken by participants suffering from nonspecific low back pain to assess the correlation between body and motion perception and perception disturbances due to pain.

\subsection{Commercial game devices in pain care}

Jointly with or beyond the use of VR for pain management, other disruptive technologies have been found beneficial to pain management with an emphasis on therapy and rehabilitation. Gaming technologies such as Microsoft Kinect and Nintendo Wii have been widely used for 'exergames', a term underpinning games for physiotherapeutic exercising, and for a broad range of neurophysiological conditions. Previous research by the authors showed promising results with engagement and effectiveness of the intervention for stroke rehabilitation using customised VR games and gaming sensors [18][25].

Accordingly, there are many opportunities for the applicability of VR and gaming technologies to pain related interventions. As such, the study of Park, Lee and Ko [19] has researched the effects of using the commercially available exercise program by Nintendo Wii for factory workers with low back pain. The results of this study showed improvement both in physical and cognitive conditions. Another study illustrated the importance of physical exercise for chronic pain and also the opportunities for disruptive technologies, such as VR and gaming technologies, for designing interventions to help sufferers engage with and adhere to a physical exercise regime [21]. A very recent study by Boudreau et al. [2] discusses the reliability between the conventional 2D paper drawing for pain assessment and a 3D body schema. The authors conclude that the two interventions offer similar results in terms of reliability, with the $3 \mathrm{D}$ platform presenting with slight variant size of the area drawn. The authors remark that there is a necessity for further investigation into the effectiveness of $3 \mathrm{D}$ platforms for pain assessment.

Table 1. The use of VR and commercial game devices in pain management

\begin{tabular}{|c|c|c|c|}
\hline Author & $\begin{array}{c}\text { Interv } \\
\text { ention }\end{array}$ & Application & Benefits \\
\hline Hoffman et al [6] & VR & Burn pain & $\begin{array}{c}\text { Reduce } \\
\text { distress }\end{array}$ \\
\hline Kwekkeboom et al [9] & VR & Cancer pain & Distraction \\
\hline $\begin{array}{c}\text { Trost \& Parsons [24]; } \\
\text { Gromala et al [3]; } \\
\text { Roosink et al [20]; } \\
\text { Park et al [19]; } \\
\text { Singh et al [21] }\end{array}$ & $\begin{array}{c}\text { Kinect } \\
\text { Wii }\end{array}$ & Chronic pain & $\begin{array}{c}\text { Physical } \\
\text { exercise, } \\
\text { physical and } \\
\text { cognitive } \\
\text { conditions, } \\
\text { distraction, } \\
\text { pain-related } \\
\text { fear }\end{array}$ \\
\hline $\begin{array}{c}\text { Lamont et al [10]; } \\
\text { Guo et al [4] }\end{array}$ & VR & Limb pain & \begin{tabular}{c} 
Distraction \\
\hline
\end{tabular} \\
\hline \multicolumn{2}{|c|}{}
\end{tabular}

The above studies demonstrate the importance of VR and commercial game devices in the management of pain. However, in most cases, this was used as either a distraction technique, or as a mechanism to improve the rehabilitation process and distress (Table 1). To the best of our knowledge, there is limited, if any, research that attempts to identify the potential of commercial game devices as a tool to support the clinical assessment of this notoriously difficult experience. Accordingly, the objective of this paper is to propose a conceptual design of an interactive tool that is exploiting the usefulness and capabilities of Microsoft Kinect towards the assessment of the pain experience.

\section{DESIGN AND ARCHITECTURE}

This section introduces the concept of our conceptual model for pain modelling, registration and interaction. Firstly, we introduce the conceptual model, and secondly explain the application interaction model through Microsoft Kinect.

\subsection{Conceptual Design}

This section introduces the concept of our conceptual model for pain modelling, registration and interaction. Based on our previous results [22], we have continued to build a cloud based platform model for pain assessment and registration. It is important for such a tool to adapt to the user's context and situation and not the other way around enforcing new routines. In the background section we highlighted the new ways of interaction and communication important to our user group.

Possibilities made available through technologies such as VR, mobile and cloud heavily influence users' daily activities. Contemporary users are now converging towards an expectation to be able to interact from any device, at any place and at any time. For this reason, it is important to see the whole picture in 
terms of devices interacted with, used and exposed for during a day. Our conceptual design envisions a cloud based user profile as the hub of the system. Each user will have a global, online, profile where all the user's data and interaction is stored. The user will interact from the device of his/her choice, at the place most convenient and at the best possible time. Figure 1 illustrates the overall concept.

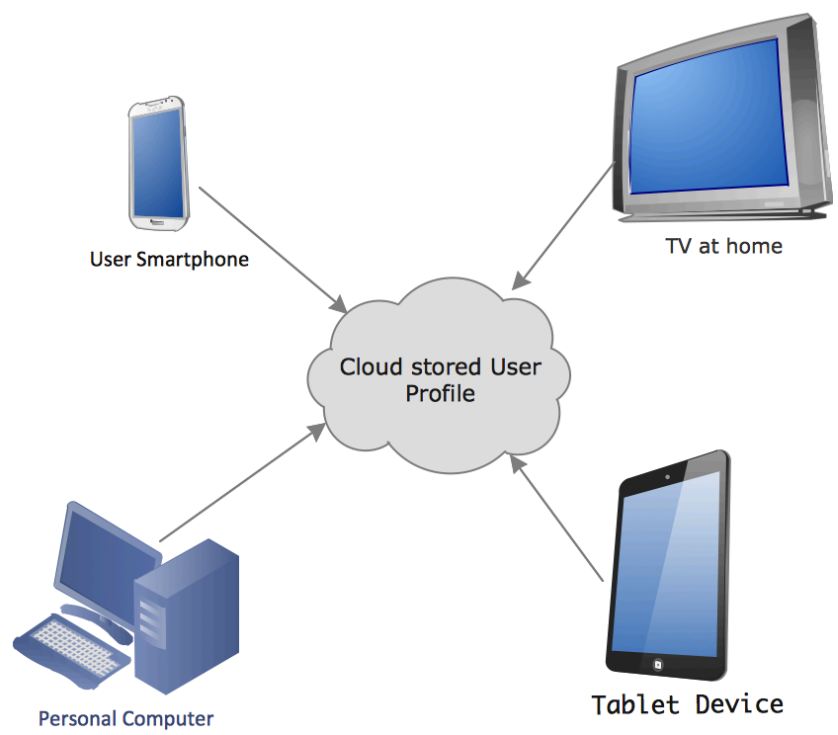

Figure 1. Conceptual Model

As detailed in the above figure, the user can interact from any preferred device. This leads to a device-independent input model for the user and seamless sharing of data across all his/her devices. The actual logging of pain level happens in a pain diary entry and each entry consists of a written description together with a 3D model visualizing the entry. The data is entered and shared through the cloud databank, making all data available on all devices and platforms. This device agnostic approach facilitates seamless online/offline environments, where offline contents will be synchronized at each occasion the device gets online, and selected online contents will equally be synchronized with local device contents.

\subsection{Application Interaction Model}

As previously introduced, the Microsoft Kinect tool is used as the tool when leveraging VR techniques for entering information into the platform. Exploiting the benefit of a large (42" +) TV screen for input, the user interface is adapted to this visualization surface (Figure 2).

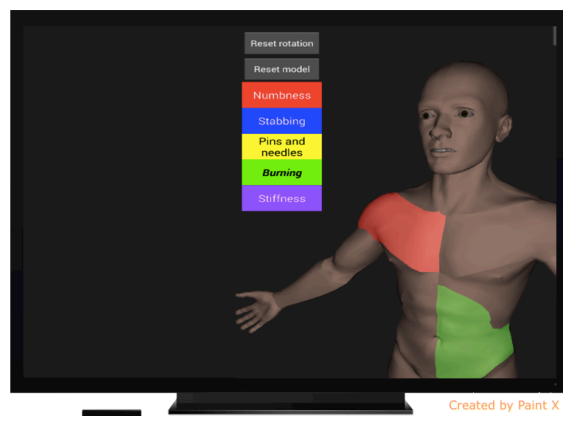

Figure 2. TV user interface for pain assessment
The user will first select the given pain type (numbness, stabbing, pins and needles, burning or stiffness) and then apply it to the mannequin by selecting the appropriate section of the body where pain is present. A visual identification by the colour of the pain category will be revealed and multi-selecting the same area more than one time will intensify the colour ( 3 levels of intensity) to indicate a stronger pain.

The novel interaction model of Microsoft Kinect enables unique and diverse possibilities. The user, positioned in front of the TV, will use gestures to interact with the application and manipulate the mannequin (Figure 3). For users with full mobility, a wide range of interaction gestures are available, facilitating a detailed interaction level. For users with reduced or low mobility, a stripped down version, with less complicated gestures will be present. Further to this, for users with close to no mobility, VR headsets are applied to reduce the threshold and complexity for system interaction and pain assessment.

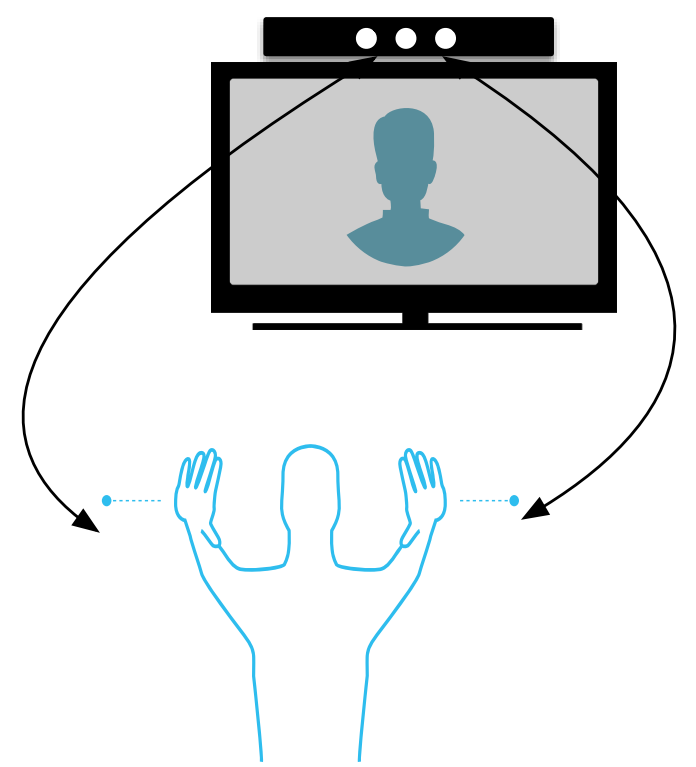

Figure 3. Pain assessment through Kinect interaction

\section{CONCLUDING DISCUSSION}

This paper presents the conceptual model and the interaction method behind a tool that will be used towards the assessment of the pain experience. As opposed to our previous work [22], which concentrates on a platform-specific approach, in this paper we propose a platform-agnostic architecture that takes into consideration that modern users employ a wide variety of devices to access cloud based content.

Considering the numerous possibilities that the above paradigm suggests, the proposed tool will leverage the popularity of bespoke gaming sensors, such as Microsoft Kinect, in order to provide a more naturalistic experience when interacting with cloud based content via a TV user interface.

It is anticipated that this tool could be a promising intervention for use both in the clinical and home environment.

Future work is in plan to address the development and evaluation of the proposed tool with a sample of participants with various types of pain. 


\section{REFERENCES}

[1] Baker, M. et al. 2010. Improving the current and future management of chronic pain: A European Consensus Report. http://www.pfizer.co.uk/sites/PfizerCoUK/Media/Documents $/$ Pain\%20Proposal\%20-

\%20European\%20Consensus\%20Report.pdf. Accessed: 2016-02-12.

[2] Boudreau, SA. et al. 2016. Digital Pain Drawings: Assessing Touch-Screen Technology and 3D Body Schemas. Clin J Pain. 32, 2, 139-45.

[3] Gromala, D. et al. 2015. The Virtual Meditative Walk. 33rd Annual ACM Conference on Human Factors in Computing Systems, 521-524.

[4] Guo, C., Deng, H., Yang, J. 2015. Effect of virtual reality distraction on pain among patients with hand injury undergoing dressing change. J Clin Nurs, 24(1-2), 115-120.

[5] Haefeli, M. Elfering, A. 2006. Pain assessment. Eur Spine J $15,17-24$.

[6] Hoffman, HG. et al. 2011. Virtual reality as an adjunctive non-pharmacologic analgesic for acute burn pain during medical procedures. Ann Behav Med 41, 183-191.

[7] IASP International Association for the Study of Pain. 2012. http://www.iasppain.org/Taxonomy?navItemNumber=576\#Pain. Accessed: 2016- 02- 12 .

[8] Jamison, RN. Fanciullo, GJ. Baird, JC. 2004. Usefulness of Pain Drawings in Identifying Real or Imagined Pain: Accuracy of Pain Professionals, Non-professionals, and a Decision Model. J Pain 5, 476-482.

[9] Kwekkeboom, KL. et al. 2010. Mind-body treatments for the pain-fatigue-sleep disturbance symptom cluster in persons with cancer. J Pain Symp Manag 39, 126-138.

[10] Lamont, K. Chin, M. Kogan, M. 2011. Mirror box therapy: seeing is believing. Explore 7, 369-372.

[11] Lee, SJ. 2001. Pain measurement: Understanding existing tools and their application in the emergency department. Emerg Med 13, 279-287.

[12] Malliou, P. Gioftsidou, A. Beneka, A. Godolias, G. 2005. Measurements and evaluations in low back pain patients. Scand J Med Sci Sports 16, 219-230.

[13] Mannion, AF. Balague, F. Pellise, F. Cedraschi, C. 2007. Pain measurement in patients with low back pain. Nat Clin Pract Rheumatol 3 (11), 610-618.

[14] Mäntyselkä, P. et al. 2002. Direct and indirect costs of managing patients with musculoskeletal pain-challenge for health care. Eur J Pain. 6, 2, 141-148.
[15] Masferrer, R. Prendergast, V. Hagell, P. 2003. Colored pain drawings: preliminary observations in neurosurgical practice. Eur J Pain 7, 213-217.

[16] Ohnmeiss, DD. 2000. Repeatability of Pain Drawings in a Low Back Pain Population. Spine 25, 980-988.

[17] Paraskevopoulos, I., Tsekleves, E. 2013. Use of Gaming Sensors and Customised ExerGames for Parkinson's Disease Rehabilitation. 5th International Conference on Games and Virtual Worlds for Serious Applications, 1-5.

[18] Paraskevopoulos, IT. et al. 2014. Design guidelines for developing customised serious games for Parkinson's Disease rehabilitation using bespoke game sensors. Entertainment Computing, 5, 4, 413-424.

[19] Park, JH, Lee, SH, Ko, DS. 2013. The Effects of the Nintendo Wii Exercise Program on Chronic Work-related Low Back Pain in Industrial Workers. J Phys Ther Sci, 25, 8, 985-8.

[20] Roosink, M. et al. 2015. Assessing the perception of trunk movements in military personnel with chronic non-specific low back pain using a virtual mirror. PLoS One, 10, 3, 1-14.

[21] Singh, A. et al. 2014. Motivating People with Chronic pain to do Physical Activity: Opportunities for Technology Design. 32 nd annual ACM conference on Human factors in Computing Systems, 2803-2812.

[22] Spyridonis F. Hansen J. Gronli, T-M. Ghinea G. 2014. PainDroid: An Android-based Virtual Reality Application for Pain Assessment. Multim Tools Appl 72 (1), 191-206.

[23] St. John, M. Cowen, MB. Smallman, HS Oonk, HM. 2001. The Use of 2D and 3D Displays for Shape-Understanding versus Relative Position Tasks. Hum Factors 43 (1), 79-98.

[24] Trost, Z, Parsons, TD. 2014. Virtual reality graded exposure therapy as treatment for pain-related fear and disability in chronic pain. Intelligent Systems Reference Library, 68, 523546.

[25] Tsekleves, E. et al. 2014. The use of the Nintendo Wii in motor rehabilitation for virtual reality interventions: a literature review. Virtual, Augmented Reality and Serious Games for Healthcare 1, Springer-Verlag, 321-344.

[26] Tsekleves, E. et al. 2011. Wii your health: a low-cost wireless system for home rehabilitation after stroke using Wii remotes with its expansions and blender. $8^{\text {th }}$ IASTED International Conference on Biomedical Engineering.

[27] Wenig CM. et al. 2008. Costs of back pain in Germany. Eur $J$ Pain 13, 280-286.

[28] Werner, P. et al. 2013. Towards pain monitoring: Facial expression, head pose, a new database, an automatic system and remaining challenges. Proceedings of the British Machine Vision Conference, 111-119. 